\title{
Power supply network design: a case study driven approach
}

\author{
M. Eireiner ${ }^{1}$, S. Henzler ${ }^{2}$, T. Missal ${ }^{1}$, J. Berthold ${ }^{2}$, and D. Schmitt-Landsiedel ${ }^{1}$ \\ ${ }^{1}$ Institute for Technical Electronics, Technical University Munich, Germany \\ ${ }^{2}$ Infineon Technologies AG, Munich, Germany
}

\begin{abstract}
A study, based on product related scenarios, on power supply integrity issues is conducted. The effectiveness of specific design parameters depends strongly on the expected loading of the power distribution grid. Therefore, the commonly used approach to only use an even current distribution can lead to non-optimal power grid designs. For power grid optimization, a problem reduction from quadratic to linear order is presented. Simulations in a System-on-Chip (SoC) environment show, that power supply integrity mainly depends on the placing of the cores within the SoC die.
\end{abstract}

\section{Introduction}

With decreasing feature sizes, power supply integrity has become a serious concern in integrated circuit design. Lowered supply voltages, increasing current densities, increasing operating frequencies, and increasing sheet resistances have decreased the noise margins for every process technology. This trend is expected to continue with technology scaling (Nassif and Fakhouri, 2002, Larsson, 1999, Mezhiba and Friedman, 2004).

Up to now, the design of power distribution networks has been the only design method to combat power supply distortions. The design of a power distribution grids includes the choice of how many of the available metal layers are used for power distribution, as well as the sizing, in terms of widths and pitches, of the used metal layers. The thickness of a metal layer is given by technology and therefore is no design parameter.

The rest of the paper is organized as follows. In Sect. 2 and Sect. 3 the movitation and the simulation setup are outlined. Simulation results for single parameter sweeps are presented in Sect. 4, for combined parameter sweeps in Sect. 5. A SoC environment is analyzed in Sect. 6.

Correspondence to: $\mathrm{M}$. Eireiner

(eireiner@tum.de)

\section{Motivation}

Based on the International Technology Roadmap for Semiconductors (ITRS) (ITRS, 2005), for technology nodes from $130 \mathrm{~nm}$ to $45 \mathrm{~nm}$, values for sheet resistance $\left(R_{\square}\right)$ of local and intermediate signal wires, supply voltage $\left(V_{D D}\right)$, power density (P/A), current density (I/A), and the resulting IRDrop are displayed in Table 1. All values are normalized to their corresponding value in the $90 \mathrm{~nm}$ technology node. We see that from the $90 \mathrm{~nm}$ to the $45 \mathrm{~nm}$ technology, the sheet resistance will increase by a factor of 2.07 and 1.77 for local and intermediate signal wires, respectively. The current density will also increase by a factor of 1.46 . This together results in an IR-Drop increase of about a factor of 2.6-3.0 depending on the usage of local and intermediate signal wires for supply voltage delivery.

Therefore, power supply integrity is a growing concern not only for high performance, but also for low power designs.

Another point which increases the challenge of power supply network design is that the power supply network has to be designed at an early stage in the design process. Therefore, only little is know about the actual power distribution within the chip (Benoit et al., 1998). Since after the routing the power grid hardly can be changed, it is a common approach to estimate the power consumption of a chip, multiply it by a factor of three to seven, depending on the circuit architecture and on how conservative the chip is designed, and distribute it evenly over the entire chip based on a DC simulation (Dharchoudhury et al., 1998). For these reasons, power distribution grids are always designed over conservative. Therefore, up to one third of the available metal resources in one layer might be used for power distribution.

A DC simulation is much faster compared to a transient simulation. However, this comes at the cost that transient effects are lost in the simulation setup. In (Saint-Laurent and Swaminathan, 2004) it was shown that the cycle average of the supply voltage can be taken as key metric for path delay degradation. To verify this, we did simulations on a critical path replica in a $90 \mathrm{~nm}$ technology. The nominal supply voltage is $1.2 \mathrm{~V}$. In Fig. 1 the resulting path delay versus the cycle average of the variation of effective supply voltage

Published by Copernicus Publications on behalf of the URSI Landesausschuss in der Bundesrepublik Deutschland e.V. 
Table 1. Scaling properties of IR-Drop normalized to $90 \mathrm{~nm}$ technology node (ITRS, 2005).

\begin{tabular}{lcccc}
\hline & $130 \mathrm{~nm}$ & $90 \mathrm{~nm}$ & $65 \mathrm{~nm}$ & $45 \mathrm{~nm}$ \\
\hline$R_{\square}$ (local) & 0.7 & 1 & 1.54 & 2.07 \\
$\quad$ (intermed.) & 0.85 & 1 & 1.44 & 1.77 \\
$V_{D D}$ & 1.13 & 1 & 1 & 0.9 \\
$\mathrm{P} / \mathrm{A}$ & 0.87 & 1 & 1.14 & 1.31 \\
$\mathrm{I} / \mathrm{A}$ & 0.77 & 1 & 1.14 & 1.46 \\
IR-Drop & $0.54-0.65$ & 1 & $1.64-1.76$ & $2.58-3.02$ \\
\hline
\end{tabular}

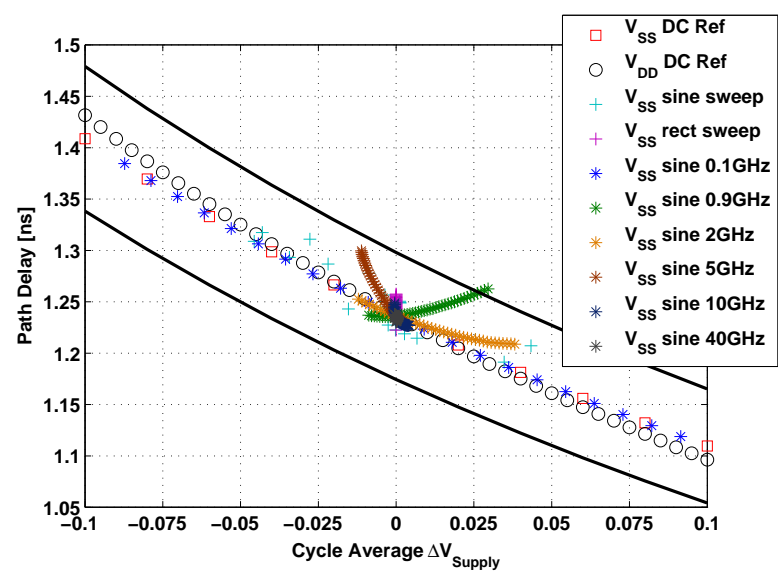

Fig. 1. Path delay versus cycle average of effective supply voltage $V_{\text {Supply for varying }} V_{S S} / V_{D D}$ curves, with amplitudes $+/-0.3 \mathrm{~V}$. The solid black lines give a $5 \%$ boundary around the $V_{S S}$ reference case.

$\Delta V_{\text {Supply }}$ is displayed. Here, the effective supply voltage $V_{\text {Supply }}$ is defined as the difference between the cycle average of $V_{D D}$ and $V_{S S}$, respectively. In turn $\Delta V_{\text {Supply }}$ is defined as the difference between the case of no noise, $1.2 \mathrm{~V}$, and the noisy one: $\Delta V_{\text {Supply }}=1.2 \mathrm{~V}-V_{\text {Supply noise. }}$ The graphs with the circles and rectangular symbols are simulation sweeps, in which $V_{D D}$ and $V_{S S}$, respectively, are kept constant during one simulation run. Both graphs act as reference. The solid black lines give a $5 \%$ boundary around the $V_{S S}$ sweep. The graphs with crosses and stars show the results of simulations with transient varying $V_{S S}$ curves. Sinodial and rectangular curves were taken with varying amplitude and/or frequency. The frequency range for the sinodial noise was swept from $100 \mathrm{MHz}$ up to $40 \mathrm{GHz}$. However, most of the simulation results, which had almost perfect correlation with the reference simulation are not shown due to reasons of clarity. The peak to peak amplitude of the ground disturbance was up to $0.6 \mathrm{~V}$. We see that all simulations, with one minor exception, show an error which is well below 5\%. Simulations with transient varying $V_{D D}$ curves showed similar results. Therefore, the

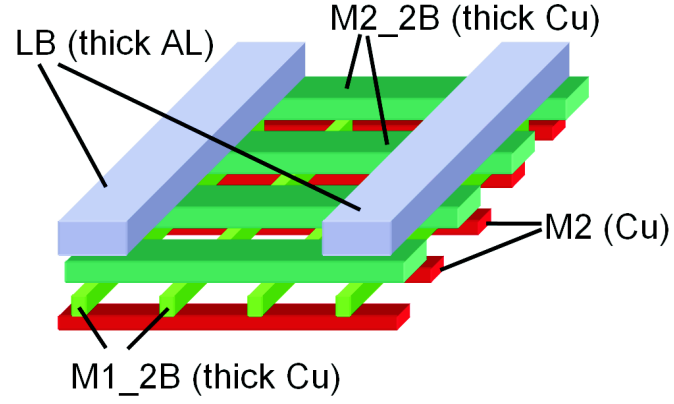

Fig. 2. Schematic of the used 4 stages power grid.

exact waveform of $V_{D D}$ and $V_{S S}$ are not of concern but only their cycle average has to be taken into consideration if the effect of power supply distortions on path delay variations is analyzed. Hence, fast DC simulations instead of expensive transient simulations were performed to allow for a fast evaluation of varying power grid sizings.

\section{Simulation setup}

We base our analysis on the example of an ARM926 core in a 90nm technology, as it is described in (Lueftner et al., 2006). As initial point of our analysis we take a power distribution grid, which consists of four layers. The top layer, LB, is a thick aluminum layer, followed by two copper metal layers with double height for intermediate signal wiring, M2_2B and M1 $2 \mathrm{~B}$, and finally the local power rails in the M2 layer, which is a copper layer with single height. The layers are orthogonal to each other and are connected frequent with vias at the crossing points. The schematic is depicted in Fig. 2 . For our analysis we take a symmetric $600 \mu \mathrm{m} \times 1200 \mu \mathrm{m} \mathrm{sec}-$ tion of the power grid. The end points of the layers at the section boundary are short-circuited with their starting points to supress artefacts.

From the power data given in (Lueftner et al., 2006) the average power consumption was taken and multiplied by a factor of three for temporal variations. For lateral variations of the grid loading, two different settings were analyzed. In the first loading scenario the power is distributed evenly across the whole chip. In the second scenario, a hotspot was created in the middle of the power grid. The hotspot is $150 \mu \mathrm{m} \times 300 \mu \mathrm{m}$ and has a current density which is three times higher than in the case of the even loading, the schematic is depicted in Fig. 3. The current density in the rest of the chip was chosen such that for Even and Hotspot loading the same total current density resulted. 


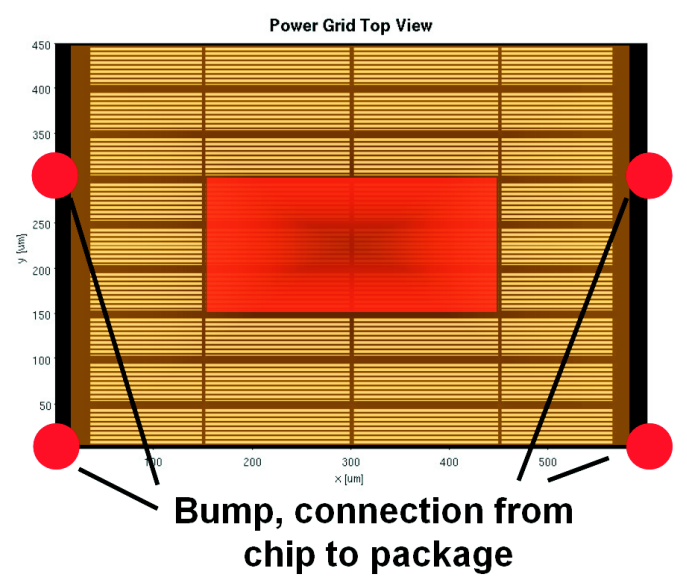

Fig. 3. Schematic of the hotspot loading. A hotspot, $150 \mu \mathrm{m} \times 300 \mu \mathrm{m}$, with a three times higher current density compared to the even case is placed in the middle of the power grid. The remaining power grid was loaded such that the overall current density matched for Hotspot and Even loading.

\section{Single parameter sweeps}

In the first analysis all design parameter of the power grid are varied one at the time, with the others at their initial value, and the resulting IR-Drop was simulated for both loading scenarios. The pitch of the lowest power rail is given through the height of a standard cell. The pitch of the LB layer is set through minimum bump pitch requirements. Therefore, six independent design parameters, four width and two pitch parameters, exist.

For every design parameter a sweep across a wide parameter range was conducted. The increase or decrease of a design parameter was translated into an equivalent relative increase or decrease in area. The resulting IR-Drop depending on the change in area is displayed in Fig. 4, here at the example of varying M2 width with Hotspot loading. The resulting graphs for the remaining five design parameters look similar to the one for the M2 width sweep in Fig. 4. Changing from Hotspot to Even loading results in a decrease of IRDrop by preserving roughly the shape of the graph. However, it has to be mentioned that in our use case, in which the grid is connected by bumps to the package supply every $600 \mu \mathrm{m}$ see Fig. 2, virtually no voltage drop occurs in the LB layer. Therefore, changing the width of the LB layer has minimal impact on the resulting IR-Drop.

Based on the simulations described above, a cost performance analysis in terms of resulting change in IR-Drop and change in occupied area is conducted for both loading scenarios. Fig. 5 shows the effectiveness of a design change for the different design parameters. Effectiveness is defined as $\frac{\Delta \mathrm{IR}-\text { Drop }}{\Delta \text { rel.area }}$. The effectiveness is derived from the criterion how much area is needed to achieve a given reduction in IR-

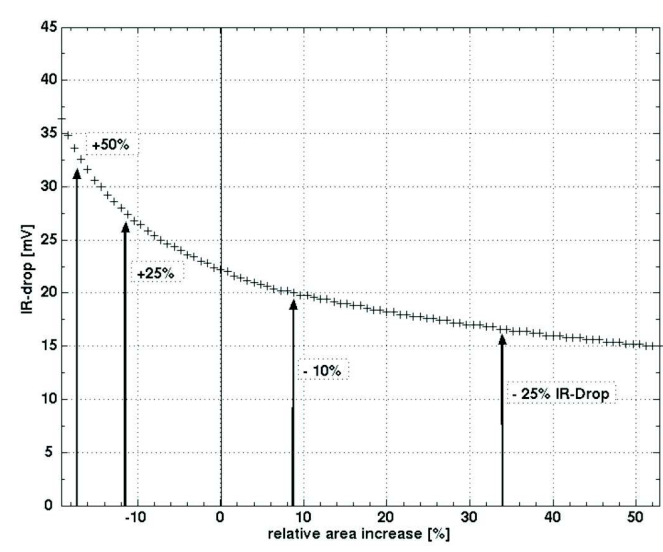

Fig. 4. Resulting IR-Drop for varying M2 width for the Hotspot loading. An increase/decrease of M2 width is translated into a relative increase/decrease of area.

Drop. The less area needed, the more efficient the design parameter is. The results in Fig. 5 are normalized to the maximum effectiveness in one loading scenario. The LB layer is omitted in this plot, since, as mentioned before, it does not contribute significantly to IR-Drop.

We see that for different loading scenarios of the power grid, the effectiveness of the design parameter drastically changes. For example for Even loading M1_2B pitch has the lowest effectiveness, whereas for a Hotspot loading the situation is vice versa, M1_2B pitch is the preferable parameter to reduce IR-Drop area efficient.

The loading of an actual design hardly will be an Even loading, and the critical case for IR-Drop is some hotspot on the chip. Therefore, we see that the common approach to use Even loading for initial power grid dimensioning gives wrong design incentives. This can lead to non-optimal power grid designs in terms of area efficiency. Therefore, we propose to use not only Even loading, but also some Hotspot loading for initial power grid design. With the additional loading scenario effects of lateral varying current densities on power grid dimensioning can be accounted for. Hence, right from the start a better power grid design is possible, which helps to achieve the area efficient optimum.

\section{Combined parameter sweeps}

Power grid design usually is not done by only varying one design parameter, but multiple at a time. The question we want to address in this section is, whether it is possible to estimate the resulting IR-Drop of varying two design paramters at the same time, by taking the simulation results of their single parameter sweeps. Therefore, it would be possible to achieve a problem reduction from a quadratic order, $O\left(N^{2}\right)$, 


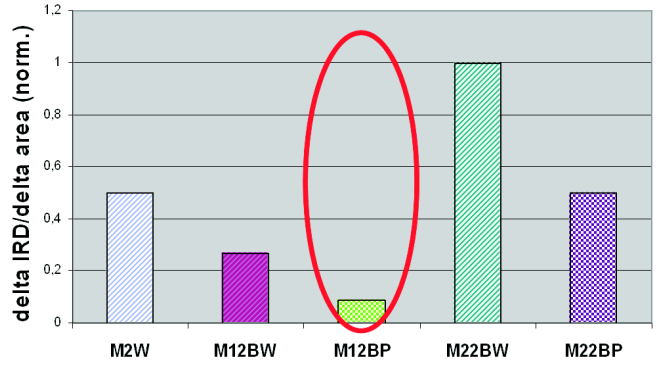

a)

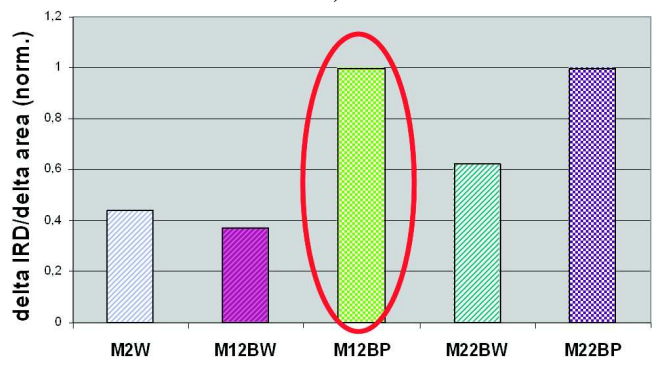

b)

Fig. 5. Effectiveness in terms of IR-Drop over relative area change for given reduction in IR-Drop for varying design paramters. (a) Even loading (b) hotspot loading

to linear order, $O(N)$. This in turn decreases the simulation time signficantly.

First we tried to estimate the resulting IR-Drop with the additive equation:

$\operatorname{IRD}_{\text {est }}\left(v_{1}+\Delta v_{1}, v_{2}+\Delta v_{2}\right)=\operatorname{IRD}_{\text {single }}\left(v_{1}+\Delta v_{1}\right)$

$+\operatorname{IRD}_{\text {single }}\left(v_{2}+\Delta v_{2}\right)-\mathrm{IRD}_{\text {std }}$

Where $\operatorname{IRD}_{\text {est }}\left(v_{1}+\Delta v_{1}, v_{2}+\Delta v_{2}\right)$ is the estimated IR-Drop for changing design paramters $v_{1}$ and $v_{2}$, $\Delta \operatorname{IRD}_{\text {single }}\left(v_{1}+\Delta v_{1}\right)$ and $\Delta \operatorname{IRD}_{\text {single }}\left(v_{2}+\Delta v_{2}\right)$ are the changes in IR-Drop caused by single design paramter variation $\Delta v_{1}$ and $\Delta v_{2}$, respectively, $I R D_{\text {std }}$ is the IR-Drop for the initial or standard dimensioning of the power grid. As reference all combinations of parameter sweeps were simulated. However, the additive IR-Drop estimation showed high deviation from the reference simulation and therefore can not be used for an acurate estimation.

As a second approach we tried to estimate the resulting IR-Drop with the multiplicative equation:

$$
\begin{aligned}
& \operatorname{IRD}_{\text {est }}\left(v_{1}+\Delta v_{1}, v_{2}+\Delta v_{2}\right)= \\
& \frac{\operatorname{IRD}_{\text {single }}\left(v_{1}+\Delta v_{1}\right) \cdot \operatorname{IRD}_{\text {single }}\left(v_{2}+\Delta v_{2}\right)}{\operatorname{IRD}_{\text {std }}}
\end{aligned}
$$

To visualize the normalized error, Err $_{\text {norm }}$, between the simulated and the estimated IR-Drop the error was calculated for all parameter cominations:

$\mathrm{Err}_{\text {norm }}=\frac{\mathrm{IRD}_{\text {sim }}-\mathrm{IRD}_{\mathrm{est}}}{\mathrm{IRD}_{\mathrm{sim}}}$

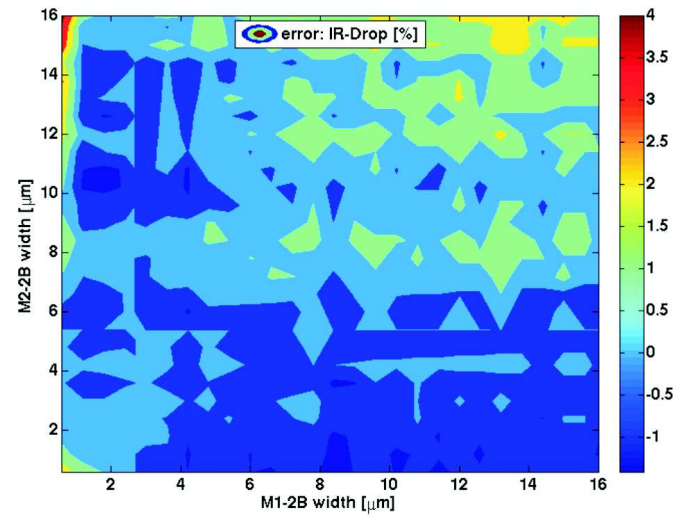

Fig. 6. Resulting error map for estimating IR-Drop multiplicatively, for varying M1_2B width and M2_2B width.

The resulting error map over the entire parameter range, here at the example for varying M1_2B width and M2_2B width, is displayed in Fig. 6. The color of the map reflects the resulting error in percentage. In Fig. 7 the histogram for the error map of Fig. 6 is displayed. The error in this case is between $-2 \%$ and $+4 \%$. For all possible combinations of parameter variations over the entire relevant design space the error is in the range of $\pm 10 \%$. Therefore, the resulting IRDrop of changing two design paramters at the same time can efficiently and accurately be estimated by the proposed equation. Hence, a problem reduction from quadratic to linear order was achieved.

Up to now, no analytical derivation for the good fit of the multiplicative approach can be given. But it is expected, that parallel connections of power rails, e.g. LB \| M1_2B and M2_2B || M2, are responsible for the malfunction of the additive as well as for the good fit of the multiplicative approach. However, more research has to be done to derive an analytical verification of the observed phenomenon.

\section{SoC power grid example}

As an other example, we consider an System on Chip (SoC) design, as it is presented in (Lueftner et al., 2006). We choose a chip size of $6 \mathrm{~mm} \times 6 \mathrm{~mm}$. The schematic of an exemplary power distribution is given in Fig. 8. In the green region in the middle of the chip, regular bump connections are available. In the outer pink region no bump connections are available due to package constraints. In the red region at the edge of the chip, the I/O connections are located and therefore, no bump connections for power distribution and no power routing in the LB metal layer is possible.

As loading we again take an ARM926 core, with a die size of $1 \mathrm{~mm} \times 1 \mathrm{~mm}$. The die is placed at varying positions within the SoC and the resulting IR-Drop is simulated. Due 


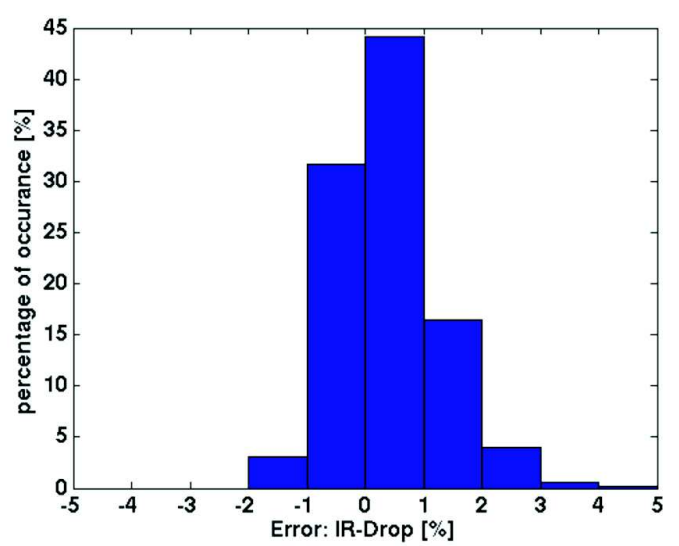

Fig. 7. Histogram for the error map of Fig. 6.

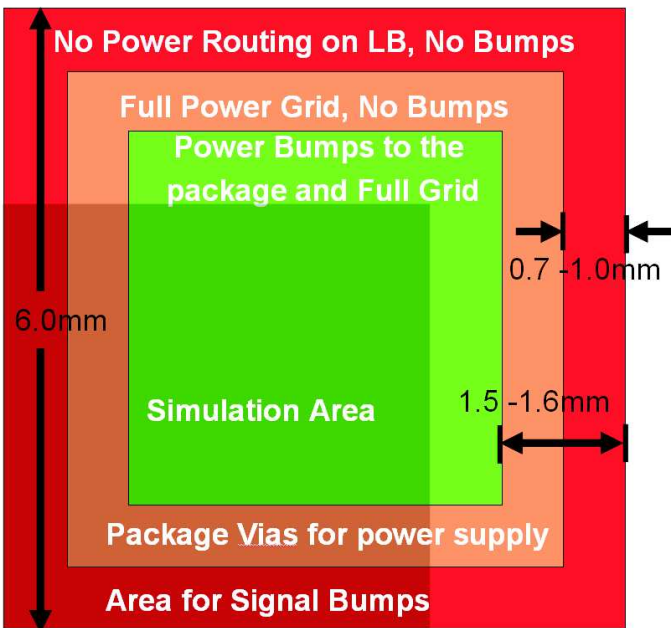

Fig. 8. Schematic of the power supply network of an example SoC with $6 \mathrm{~mm} \times 6 \mathrm{~mm}$ die size. ESD and I/O restiction prohibit the bump connections and LB usage in the outer regions of the chip.

to reasons of symmertry and to reduce simulation time the grid was reduced to the shaded area, $4 \mathrm{~mm} \times 4 \mathrm{~mm}$, in Fig. 8 . Exemplarily, three positions of the ARM core within the SoC are depicted in Fig. 9. The resulting maximum, minimum, and average IR-Drop within the core area are tabulated in Table 2. For the placing in the lower left corner, almost the entire IR-Drop is on LB and M2_2B layer. M1_2B and M2 contribute only minor to the overall IR-Drop. We see from Table 2, that by moving the core only $1 \mathrm{~mm}$ to the center, maximum IR-Drop is reduced be about a factor of 10 , for minimum and average IR-Drop the factors are even higher. Similar simulations with different placings, e.g. starting in the left center, were also conducted and showed compareable results. Therefore, in a SoC environemnt the placing of the major power consuming cores is the most critical issue for

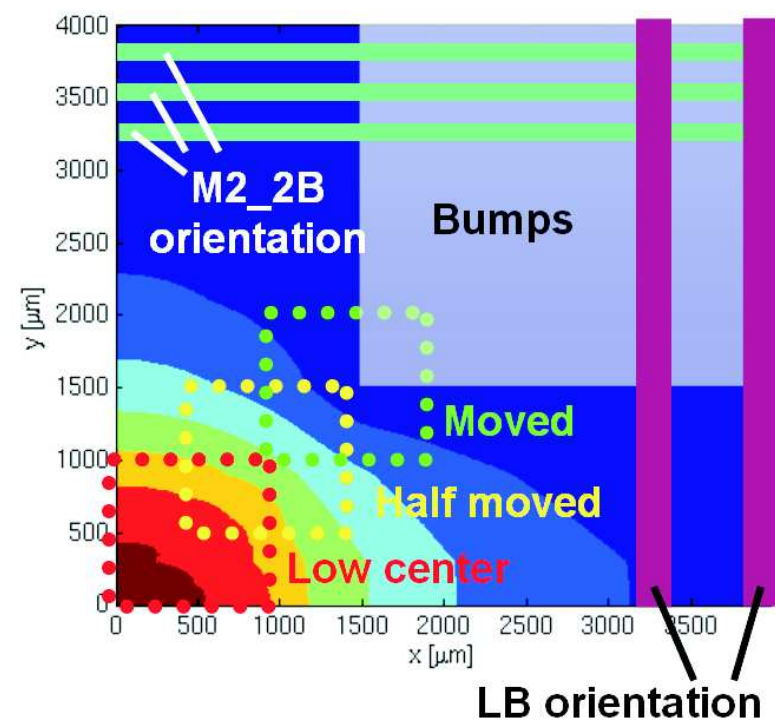

Fig. 9. Schematic of the power supply network of an example SoC with $6 \mathrm{~mm} \times 6 \mathrm{~mm}$ die size. ESD and I/O restiction prohibit the bump connections and LB usage in the outer regions of the chip.

Table 2. Power supply noise for varying core positions within SoC power grid.

\begin{tabular}{cccc}
\hline & Low center & Half moved & Moved \\
\hline Max & $126.3 \mathrm{mV}$ & $42.4 \mathrm{mV}$ & $13.1 \mathrm{mV}$ \\
Min & $96.7 \mathrm{mV}$ & $30.1 \mathrm{mV}$ & $7.2 \mathrm{mV}$ \\
Avg & $35.9 \mathrm{mV}$ & $0.2 \mathrm{mV}$ & $0.2 \mathrm{mV}$ \\
\hline
\end{tabular}

power supply integrity. However, I/O and ESD restrictions might require a placing of such blocks to the edges and I/O pins of the SoC.

\section{Conclusions}

In this paper a case study driven approach to power supply integrity analysis was presented. Based on product related scenarios, it was shown that using only the assumption of Even loading for initial power grid design can lead to nonoptimal power grids. For the optimization of power grids, it was shown that a problem reduction from quadratic to linear order is possible by using a multiplicative IR-Drop estimation. However, an analytical derivation of the used formula could not be given and is the goal of future research, as well as the extension from two to multiple design variables. In SoC environments in which $\mathrm{I} / \mathrm{O}$, packaging, and ESD requirements restrict the on-chip power routing, almost the entire IR-Drop is in the upper layers. Placing of cores 
within the SoC has the highest single impact on power supply integrity.

\section{References}

Benoit, M., Taylor, S., Overhauser, D., and Rochel, S.: Power Distribution in High-Performance Design, in: Proceedings of IEEE Symposium of Low-Power Electronics Design, 274-278, 1998.

Dharchoudhury, A., Panda, R., Blaauw, D., and Vaidyanathan, R.: Design and Analysis of Power Distribution Networks in PowerPC Mircoprocessors, in: Proceedings of Design Automation Converence, 738-743, 1998.

ITRS: International Technology Roadmap for Semiconductors, http://public.itrs.net/, 2005.

Larsson, P.: Power Supply Noise in Future IC's: A Crystal Ball Reading, in: IEEE Custom Integrated Circuits Conference, 467474, 1999.
Lueftner, T., Berthold, J., Pacha, C., Georgakos, G., Sauzon, G., Hoemke, O., Beshenar, J., Mahrla, P., Just, K., Hober, P., Henzler, S., Schmitt-Landsiedel, D., Yakovleff, A., Klein, A., Knight, R., Acharya, P., Mabrouki, H., Juhoor, G., and Sauer, M.: A 90 nm CMOS Low-Power GSM/EDGE Multimedia-Enhanced Baseband Processor with $380 \mathrm{MHz}$ ARM9 and Mixed-Signal Extensions, in: 2006 ISSCC Digest of Digital Papers, 252-253, 2006.

Mezhiba, A. V. and Friedman, E. G.: Scaling Trends of On-Chip Power Distribution Noise, IEEE Transactions on Very Large Scale Integration (VLSI) Systems, 12, 386-394, 2004.

Nassif, S. R. and Fakhouri, O.: Technology Trends in PowerGrid-Induced Noise, in: Proceedings of the 2002 international workshop on System-level interconnect prediction, 55-59, ACM Press, 2002.

Saint-Laurent, M. and Swaminathan, M.: Impact of Power-Supply Noise on Timing in High-Frequency Microprocessors, IEEE Transactions on Advanced Packaging, 27, 135-144, 2004. 